Meta

Journal des tradlucteurs

Translators' Journal

\title{
Traducteur, philosophe, poète, qui est-il ?
}

\section{Jacques Flamand}

Volume 26, numéro 4, décembre 1981

URI : https://id.erudit.org/iderudit/002746ar

DOI : https://doi.org/10.7202/002746ar

Aller au sommaire du numéro

Éditeur(s)

Les Presses de l'Université de Montréal

ISSN

0026-0452 (imprimé)

1492-1421 (numérique)

Découvrir la revue

Citer cet article

Flamand, J. (1981). Traducteur, philosophe, poète, qui est-il ? Meta, 26(4),

350-358. https://doi.org/10.7202/002746ar d'utilisation que vous pouvez consulter en ligne.

https://apropos.erudit.org/fr/usagers/politique-dutilisation/ 


\section{Traducteur, philosophe, poète, qui est-il ?*}

JACQUES Flamand

\section{LE TRADUCTEUR, LE PHILOSOPHE ET LE POÈTE}

Quel titre, quel sujet ambitieux pour le traducteur de la base, pour le traducteur ordinaire qui n'a d'autre prétention que de faire modestement son lot quotidien de traduction! Et ces trois personnages ainsi nommés - traducteur, philosophe, poète - qu'ont-ils donc en commun qu'on puisse les rassembler en une trinité qui serait au moins une famille d'esprit? Personnages séparés? Ou bien ont-ils en commun des liens fraternels que saura reconnaitre le véritable traducteur? Et si le traducteur était, presque à son insu, lui aussi un peu philosophe et un peu poète? Ce serait alors une opération réussie à laquelle je serais parvenu, je veux dire une opération de dévoilement d'une réalité obscurément vécue, modestement expérimentée au jour le jour. Car le traducteur n'est pas l'être falot et ranci dont certaines images d'Epinal délavées ont agacé nos yeux et nos esprits. Nous allons mieux le découvrir ensemble.

\section{PHILOSOPHE ET TRADUCTEUR: CONVERGENCES}

\section{S'étonner, apprendre, interroger}

Pour entrer en matière, je fais brièvement appel à un moderne et à un ancien. Le moderne, Paul Valéry : "La façon d'un philosophe, son entrée en danse est bien connue... Il esquisse le pas de l'interrogation "'. L'ancien, Platon: «[...] être avide d'apprendre et être philosophe, c'est la même chose ${ }^{2}$. «S'étonner: voilà un sentiment qui est tout à fait d'un philosophe. La philosophie n'a pas d'autre origine $\left[\ldots . . »^{3}\right.$.

Avidité d'apprendre, interroger, s'étonner. Voilà bien quelques-unes des qualités fondamentales qui font le philosophe. Le philosophe est un esprit curieux, jamais rassasié, qui ne se satisfait pas d'une première lecture du réel. Il lit et relit le même texte d'écriture ou de vie, pour découvrir la substance derrière l'accidentel, l'essentiel voilé par l'anecdotique, l'être par-delà l'apparence; ou encore, pour appliquer cette attitude à un cas plus limité, il recherche le fond derrière la forme, le message porté par les mots.

Le philosophe n'est pourtant pas un savant, ce que j'illustre par une remarque plaisante de Paul Valéry: «Le philosophe n'en sait réellement pas plus

\footnotetext{
* Conférence, Assemblée de l'Association des traducteurs et interprètes de l'Ontario, Toronto, 1980

1. Paul Valéry, La Pléiade, I, 1395.

2. Platon, République, III, $376 \mathrm{~b}$.

3. Platon, Théétète, $155 \mathrm{~d}$.
} 
que la cuisinière; si ce n'est en cuisine, où elle s'entend réellement (en général) mieux que lui.

Mais la cuisinière (en général) ne se pose point de question universelle. Ce sont donc les questions qui font le philosophe. Quant aux réponses... Par malheur, il y a dans chaque philosophe un mauvais génie qui a réponse à tout $" 4$.

Je ferais une réserve à propos de ces lignes de Valéry : aussi justes soientelles quant au fond, je trouve désobligeant et "petit bourgeois» de prétendre que la cuisinière, même «en général», ne se pose point de question universelle. Je connais des gens bien simples qui réfléchissent plus que de prétendus philosophes.

Philosopher, c'est chercher les raisons et, principalement, les raisons profondes des choses. Pourquoi? (causalité) et Pour quoi? (finalité) sont deux interrogations constantes du philosophe. Comme l'a écrit A. Cournot, «le philosophe est voué par état à chercher la raison des choses» 5 . En cela, le philosophe est méta-physicien. Par état également, il étudie les formes de la pensée et les procédés généraux de l'esprit humain ${ }^{6}$. Il exerce alors la fonction de logicien. Le philosophe veut tout expliquer, tout comprendre. Or, cette attitude d'esprit devrait être celle du traducteur, dans son travail quotidien, comme dans sa vie tout court d'homme ou de femme. J'y reviendrai. Mon propos peut paraitre ambitieux. Il n'est pas insensé.

\section{Le sens de la totalité}

Une autre qualité que j'ai à peine évoquée : le sens de la totalité et de la solidarité des êtres. J'en ferai une application à l'activité du traducteur. Car, pour moi, la fonction du traducteur n'est pas banale. «Etre philosophe, a-t-on écrit, c'est tenir compte de tout, et premièrement des expériences les plus hétérogènes aux siennes propres. Ce n'est jamais consentir à la partialité sous aucune forme" ${ }^{7}$.

$\mathrm{Ne}$ jamais être partial, tenir compte du tout, sortir de soi pour aller à l'autre. Application ici : tenir compte du tout, c'est replacer le mot dans la phrase, la phrase dans le texte, le texte dans le contexte. Traduire, c'est traduire le tout. La partie ne se comprend que par rapport à l'ensemble. Comme le philosophe, le traducteur doit avoir le sens de la solidarité, de l'interrelation des éléments.

Sortir de soi pour aller à l'autre, que cet autre soit être personnel ou non, demande des qualités d'objectivité et le sens de l'universel. Ces qualités sont celles du traducteur qui part à la découverte d'un texte, de la signification d'un texte objectif, lequel résiste à son analyse, à la lecture qu'il en fait. Le texte me dépasse, moi, traducteur. Il est extérieur à moi; il existe en soi.

4. Paul Valéry, Rhumbs, p. 193-194.

5. A. Cournot (1872): Considérations sur la marche des idées et des événements dans les temps modernes, 1,8 .

6. Cf. A. Cournot, Essai sur les fondements, par. 325, p. 479.

7. René Schérer (1961): "Le péché capital du philosophe», Les Études philosophiques, $\mathrm{n}^{\circ}$ spécial, la Nature humaine, Paris, P.U.F., p. 172. 
Je me dois de le respecter. Je dois me soumettre à une exigence de compréhension complète et de fidélité.

\section{La fidélité}

Fidélité. J'ai prononcé le mot. Il me faut donc l'expliquer, et un peu en philosophe, puisque j'ai adopté ce point de vue inusité du traducteur-philosophe.

Henry Duméry, penseur contemporain, profond et subtil, a écrit : «La vraie fidélité est création personnelle. Comprendre une doctrine, c'est accomplir le dépassement qu'elle a rendu possible. Ce n'est pas la mettre en fiches, c'est la refaire ${ }^{8}$.

Telle peut être définie la fidélité du philosophe, qui comprend et épouse une idée, une doctrine. La fidélité véritable suppose intégration, assimilation, si bien que l'extérieur devient intériorité, l'objet se fait sujet. Je fais mien l'objet et pourtant je le dépasse, je le transcende. Création personnelle, parce que je ne suis pas passif, mais acteur. J'agis l'idée, la doctrine; je la fais mienne. Elle devient une de mes convictions; elle devient moi. Aussi pourraije la réexprimer dans un langage clair et convaincant, dans une langue aisée. Je refais la doctrine, je la recrée pour moi. Si je peux, bien sûr, en faire l'analyse, la décomposer, autrement dit, la mettre en fiches, ce n'est qu'une étape de la démarche qui aboutit à la compréhension. L'étape de la mise en fiches, de l'analyse est nécessaire, mais elle doit conduire à la synthèse. Dans l'ordre de l'existence, la synthèse est action autant que réflexion. Dans l'ordre du discours, la synthèse restera théorique, notionnelle. La fidélité du traducteur dans son acte de traduction se situera habituellement au plan du discours, donc à un plan plus restreint. N'empêche que le traducteur fait acte de création quand il réinterprète; quand, par-delà le mot à mot, il réexprime le message du texte. Point de tyrannie de la forme, car la forme est au service du fond, du contenu. Le traducteur comprend le texte, il l'assimile, le repense. Il le réexprime alors dans la langue d'arrivée, avec toutes les nuances du texte original, mais peut-être avec une économie plus grande de mots, voire d'images et de métaphores. En bref, le texte d'arrivée est parfois nettement supérieur au texte de départ. Le traducteur n'est donc pas un translittérateur asservi aux mots et aux phrases. Il est interprète d'une pensée, d'un message, qu'il reformule avec autant de précision et de clarté (parfois plus même) que le texte de départ. Il fait preuve d'une double fidélité : envers l'auteur, envers le destinataire. Telle est l'œuvre de vraie fidélité du traducteur, attentif à l'esprit plus encore qu'à la lettre.

\section{Fonction réflexive et critique}

«La fonction du philosophe est réflexive et critique», a écrit Henry Duméry ${ }^{9}$. Le philosophe, par sa réflexion critique, est exégète de la vie, de

8. Henry Duméry (1956): la Tentation de faire d" bien, «Esprit», «La condition humaine», Paris, Seuil, p. 269.

9. Henry Duméry (1957): Critique et Roligion. Problèmes de méthode en philosophie de la religion, Paris, SEDES, p. 75. 
l'action. Il connaît et agit, puis réfléchit sur l'action. Il pense après coup la pensée spontanée. Ainsi, «la philosophie est pensée seconde, retour sur la pensée première qu'elle suppose: sans spontanéité, pas de réflexion. Aussi la philosophie n'est-elle pas créatrice, elle implique un donné ${ }^{10}$. De même, le traducteur, comme j'y ai déjà fait allusion, n'invente pas le texte. Il le reçoit comme un donné objectif, tout comme le philosophe reçoit le domaine sur lequel s'appliquera sa réflexion. Le traducteur exerce sa fonction de décodeur et d'interprète d'un message sur un donné - un texte - qui lui vient de l'extérieur. En ce sens, il est moins créateur que le rédacteur; il est le déchiffreur et l'interprète qui vient après. La philosophie est «méthode de discernement critique " ${ }^{11}$. Or, "le but de la critique n'est pas de dissoudre : il est de comprendre. Et comprendre n'est pas expliquer, c'est-à-dire décomposer en éléments dénombrables. Comprendre, précise Duméry, c'est saisir en homme les démarches de l'homme, intelligemment et librement, ce qui procède de l'intelligence et de la liberté ${ }^{12}$.

À l'image du philosophe, le traducteur comprend le texte, et il le comprend par le contexte. Il rend d'abord le sens du texte. S'il doit, par nécessité, traduire les phrases les unes après les autres, donc, d'une certaine manière, expliquer, décomposer, il lui faut relier les phrases les unes aux autres en un tout cohérent et un qu'est le texte. Il saisit la démarche de l'auteur du texte et la rend le plus fidèlement possible dans l'autre langue, dans l'autre culture. Il s'agit d'une fidélité au sens, d'abord, une fidélité à l'intention, non une littéralité formelle. La science du traducteur est une textologie.

La critique du philosophe est «une récupération méthodique, systématique des activités de la pensée spontanée " ${ }^{13}$. Pour le traducteur, l'équivalent de la pensée spontanée est le texte reçu du rédacteur. Le rédacteur est, en quelque sorte, le créateur. Mais le traducteur, par sa compréhension du texte et la transposition linguistique et culturelle qu'il en donne, prolonge la création originale; il est co-créateur. Comme l'écrit J.-R. Ladmiral, «la traduction est une méta-communication qui passe nécessairement par la médiation de la subjectivité du traducteur, qui fait dès lors figure d'interprète, à tous les sens du mot $\gg^{14}$. Le traducteur comprend et interprète le texte-source dans la traduction-cible qu'il en donne. Ainsi, pour mieux pénétrer un texte dense et difficile, philosophique par exemple, écrit dans sa propre langue, on a tout avantage à lire les traductions éventuelles de ce texte en langues étrangères. «Les traductions continuent ou achèvent les textes-source, dont elles sont pour ainsi dire l'exécution ${ }^{15}$. En effet, pour bien saisir une idée, il est très utile d'en avoir plusieurs formulations ou présentations. Ce peut être de la même per-

10. Jean Lacroix (1972): le Personnalisme comme anti-idéologie, "Le philosophe", «SUP», Paris, P.U.F., p. 37.

11. H. Duméry, ibid., p. 276.

12. H. Duméry (1957): Philosophie de la Religion. Essai sur la signification du christianisme, t. I, Catégorie de sujet, catégorie de grâce, Paris, P.U.F., p. IX.

13. H. Duméry, la Tentation de faire du bien, p. 140-141.

14. J.-R. Ladmiral (1979): Traduire: théorèmes pour la traduction, Paris, Petite bibliothèque Payot, p. 232.

15. Ibid., p. 232. 
sonne; ainsi le pédagogue, pour se faire clairement comprendre, redit plusieurs fois la même chose, mais de différentes façons: autrement dit, c'est-à-dire, par exemple, bref, autant de mots et locutions qui introduisent une explication, une paraphrase, une illustration, une reformulation. Et l'auditeur découvre. Ce peut être encore sous la forme originale des traductions du texte dont l'intelligence pose problème, tant il est vrai que le traducteur est interprète du texte ou de l'œuvre qu'il transpose dans sa langue d'arrivée. Le texte de départ est véritablement médiatisé par l'intelligence du traducteur-interprète, lequel exerce une fonction heuristique, qui transcende tout littéralisme mécaniciste. Ainsi, le traducteur est bien «co-auteur ou réécrivain» ${ }^{16}$. Et on peut apprécier la portée de sa tâche et l'importance de son rôle intellectuel.

Pour conclure, après l'exposé de ces convergences entre le traducteur et le philosophe, je dirais que les grandes qualités intellectuelles et critiques du philosophe sont aussi celles du traducteur, même si, par métier, celui-ci les met en œuvre moins systématiquement, contraint qu'il est d'engager trop fréquemment son talent au service de besognes utilitaires et répétitives. Peut-être plus fondamentalement, pour tout homme de réflexion, l'étonnement et l'interrogation sont deux traits d'une attitude commune devant les êtres et les choses, devant «les mots et les choses» ${ }^{17}$.

\section{POĖTE ET TRADUCTEUR : UN IDÉAL COMMUN}

Le philosophe raisonne, réfléchit, interroge, ébranle les certitudes. Le poète surprend les imaginations, jusqu'à les dérouter. E. Bersot l'a justement écrit : «Les philosophes sont ici-bas [...] pour inquiéter les esprits, comme les poètes pour inquiéter les imaginations et les cœurs. Qui ne veut que vivre tranquille peut se passer des philosophes et des poètes, mais on ne se passe pas d'eux quand on veut vivre avec dignité » ${ }^{18}$. Dignité du penseur et du créateur.

Le traducteur qui raisonne, réfléchit, interroge, suit en cela une démarche philosophique: il a une attitude de philosophe. Le traducteur qui fait sans cesse appel à sa faculté d'imagination, d'invention, de création, d'organisation du réel, s'apparente au poète. C'est précisément ces qualités que Pierre Emmanuel attribue à la poésie : «[...] la poésie vraie, qui est un mode supérieur de l'attention, une exigence rigoureuse sur la moindre flexion verbale, est d'abord faculté d'invention, de création, d'organisation de la réalité toujours découverte à nouveau $» 19$.

Le poète est un être à la fois de rigueur et d'imagination, au souffle créateur. Le poète suit une logique qui n'est pas forcément celle du philosophe. Elle bouscule la logique linéaire pour découvrir une «logique rayonnante», comme l'a appelée Yvon Bélaval, logique qui révèle la nature du lan-

16. Ibid., p. 233 ; cf. p. 22.

17. Cf. Michel Foucault (1966): les Mots et les choses. Une archéologie des sciences humaines, Paris.

18. Pierre Ernest Bersot (1886) : Essais de philosophie et de morale, Paris, I, 512.

19. Pierre Emmanuel (1973): "Pourquoi?", propos recueillis par François-Régis Bastide, Bulletin menstel d'information des Editions du Seuil, n ${ }^{\circ} 172$, novembre. 
gage, qui découvre le sens caché ou voilé des mots; des mots non séparés, mais reliés; des mots qui convergent et s'unissent. "On croit naïvement que les mots isolés ont un sens, celui qui parait s'isoler dans un dictionnaire. Or, les mots ne prennent de sens que dans un ensemble et, réciproquement, tout ensemble donne un sens aux mots, quels qu'ils soient, qu'il met en rapport $[\ldots]^{20}$.

Alors, le traducteur n'est-il pas un peu poète, qui aime et goûte les mots, qui relie les mots entre eux, leur donne sens l'un par l'autre, l'un en fonction de l'autre? Le traducteur n'est pas l'homme du dictionnaire; il n'est pas l'homme du mot isolé, dévitalisé, dépossédé du contexte. Au contraire, il cherche le sens d'un mot par rapport aux autres mots, par rapport à la phrase, au texte, au contexte. Il redonne sens et vie au mot. L'interprétation et l'expression du mot, du texte sont des actes d'intelligence, mus par une inspiration créatrice, d'autant plus féconde qu'elle est riche d'expérience et de métier. Pour le poète, un mot en évoque un autre, la partie indique le tout. Il a le sens de la réalité totale, que la logique formelle du philosophe n'arrive pas à exprimer vraiment. Le poète, par son art, par sa maîtrise du verbe et son imagination créatrice qui le conduit à inventer des images, des sens nouveaux aux mots, des mots même, le poète prolonge et, en un sens, dépasse le philosophe. Il approche les confins de l'indicible, du cosmique, du magique, du mystique.

Dans cette logique rayonnante, et on pourrait parler de translogique, de métalogique, le langage, grâce au poète, semble rendu à sa fonction magique. Grâce au dévoilement du sens qu'il opère, le poète, par la médiation des mots, nous conduit dans la sphère cachée du réel. Il nous donne un avant-goût de cette aura que l'on pressent lumineuse, où l'expérience humaine peut se vivre et s'épanouir dans ce qu'elle a de plus fondamental.

«[...] Car les poètes aussi sont une race divine, inspirée lorsqu'elle chante $[\ldots]{ }^{21}$, a écrit Platon, bien que pour lui le poète soit inférieur au philosophe.

Le traducteur qui, dans une autre langue, réécrit, interprète, transpose, réexprime le sens profond et la sensibilité, la rigueur, le mouvement et l'esthétique d'un texte, fait plus cuvre d'artiste que de linguiste, de poète que de logicien, mais toujours de penseur. «Un poème, c'est d'abord un faisceau sémantique qui envoie un sens ${ }^{22}$. De même, tout texte que le traducteur doit rendre dans la langue-cible envoie un sens. Dans son effort de traduction, il rencontre deux difficultés, deux obstacles: d'ordre culturel, d'ordre linguistique.

D'ordre culturel, pour faire passer la saisie de certaines réalités non linguistiques d'une culture dans une autre; d'ordre linguistique, pour passer des formes, souvent spécifiques d'une langue, aux formes et aux découpages différents d'une autre langue. L'obstacle culturel est en partie levé grâce aux universaux culturels qui correspondent d'une pensée, d'une conception, d'une cul-

20. Yvon Bélaval (1973) : «Poésie», Encyclopaedia Universalis, vol. 13, p. 199, c. 3.

21. Platon, les Lois, III, 682a.

22. Jean Onimus, interview de Suzanne Lafrenière, Le Droit (Ottawa), 15 mars 1975, p. 18, col. 1. 
ture à l'autre. L'obstacle linguistique se surmonte lui aussi, car les structures linguistiques se traduisent, trouvent des équivalents. Mais la difficulté est accrue, s'agissant de structures métriques, stylistiques ou poétiques.

«Le vrai problème - écrit Georges Mounin - est celui de la traduction de ces messages très particuliers que sont la littérature et la poésie». Or, «une structure n'a d'intérêt que dans la mesure où elle a une fonction, c'està-dire si elle est pertinente. Pour traduire un poème, par conséquent, le problème n'est pas de traduire forme à forme, structure à structure; ce qu'il faut traduire, c'est la ou les fonctions poétiques du texte, c'est-à-dire le ou les effets qu'il produit. C'est la poésie du texte qu'il faut traduire, et non sa forme ou bien sa forme dans la mesure où l'on peut montrer qu'elle est liée à un effet ${ }^{23}$.

Pour traduire des textes littéraires ou poétiques, le traducteur doit être luimême, ou du moins un peu, écrivain et poète. A la fidélité servile, à la lettre, il préférera la fidélité profonde, l'esprit. Au lieu du mot à mot, il choisira les «belles infidèles», pour reprendre Georges Mounin. Ou, pour le dire en termes plus savants, ceux de Eugène $\mathrm{A}$. Nida ${ }^{24}$, par exemple, le bon traducteur recourra, non à «l'équivalence formelle», mais à l' "équivalence dynamique». Bref, c'est retrouver ce que je disais plus haut: pas de tyrannie de la forme qui ferait écran au sens, à la communication qu'est tout acte de traduction.

Ces qualités du poète que, hardiment, $\mathrm{j}$ 'attribue au traducteur, s'exercent à plein dans la traduction proprement poétique ou, plus généralement littéraire. Mais, et je le dis nettement, le traducteur de textes non littéraires doit posséder ces mêmes dons et qualités, s'il entend maitriser sa traduction, même si, dans son travail quotidien, il ne les met que partiellement en oeuvre.

On l'aura constaté, j'ai, pour les besoins de l'analyse, isolé des qualités qui ne sont pas séparées. Un être humain n'est pas que philosophe. Même s'il l'est beaucoup, il sera aussi un petit peu poète, et aura d'autres visages encore. Et si le traducteur unifiait en sa personne cette bipolarité humaine?

\section{PHILOSOPHE, POÈTE, TRADUCTEUR OU TROIS EN UN}

Est-il une conclusion, au terme de l'esquisse de cette double convergence philosophe-traducteur, d'une part, poète-traducteur, de l'autre? je veux dire une conclusion un tant soit peu pertinente pour le traducteur de première ligne, dont nous sommes? Oui, assurément, et c'est une conclusion que je trouve réconfortante: le traducteur possède plusieurs des qualités de rigueur et d'imagination qui font le philosophe et le poète. Et nul besoin pour lui d'aller chercher ses titres de noblesse. Sa fonction de traducteur les rassemble: homme ou femme de science; homme ou femme de l'art et, surtout, homme ou femme de culture. Le traducteur, par sa curiosité, grâce à son esprit «en point d'interrogation», s'intéresse à tout, ou presque. Ses connaissances sont nombreuses et variées. Spécialiste du maniement de la langue, il est un des rares, parmi tous les spécialistes, à avoir, par nécessité, une culture générale. Il n'a peutêtre pas de doctorat en arts plastiques du XVIIIe siècle, mais il sait parler du

23. Georges Mounin (1973) : «Traduction», Encyclopaedia Universalis, vol. 16, p. 233, col. 2.

24. Cf. E.A. Nida (1964): Toward a Science of Translating, Leyde, Brill. 
XVIII et du XVIIe et du XIXe. Il n'est d'ailleurs qu'à connaître d'un peu près le milieu des traducteurs. Bien sûr, certains n'échappent pas à la mesquinerie, mais on rencontre parmi eux nombre d'esprits originaux, cultivés, créateurs. Une culture acquise progressivement mais réelle, une culture dont vit quotidiennement le traducteur et qui fait sa richesse et son inventivité, une culture qui féconde son dynamisme et sa créativité. Avec Yvon Bélaval, je dirai que «la spontanéité, livrée à elle-même, reste pauvre; elle répète, elle a ses poncifs... La spontanéité vraiment créatrice s'acquiert avec une culture» ${ }^{25}$. Le traducteur possède cette spontanéité créatrice assise sur une large culture.

Parvenu à cette compétence et à cette sensibilité, le traducteur n'a pas de mal à bien traduire. La traduction lui devient souvent un jeu, mais un jeu maîtrisé, où il se sent à l'aise et capable de rendre le texte de départ dans une langue à la fois juste et forte. Une fois qu'il s'est imprégné du texte de départ au point de l'avoir assimilé, ce qui suppose des qualités d'analyse et d'empathie, le traducteur doit prendre, à son égard, le maximum de recul. Comme le philosophe, il sait se détacher de ses propres idées et conceptions. Dominant le texte-source par la compréhension qu'il en a (structure et composition, sens), il ne cherchera pas à traduire des mots isolés, mais des unités de discours: proposition, phrase, paragraphe et, finalement, le texte saisi comme totalité. Chacune de ces unités correspond à une unité de sens, l'unité la plus large étant évidemment le texte complet. En outre, la qualité, le rendu de sa traduction seront fonction de son imagination et de la richesse de la palette d'équivalents dont il dispose dans la langue d'arrivée; bref, de sa réserve de références culturelles, situationnelles, conceptuelles et rédactionnelles ${ }^{26}$. Autrement dit, de sa culture. Chaque formulation possible à laquelle il pensera sera passée au crible de l'autocritique, attitude philosophique par excellence. Dès lors, "on peut dire que c'est ce va-et-vient constant entre, d'une part, les déclenchements successifs de projets de formulation, de cheminements, d'orientations, de solutions plus ou moins fécondes et, d'autre part, l'esprit critique en permanence à l'affût, qui semble constituer le cceur de l'acte traductionnel» ${ }^{27}$. On le constate, le processus mental est analogue à celui de l'écrivain et du poète: imagination créatrice et analyse critique. Le traducteur met constamment en cuvre les qualités du poète et du philosophe, quels que soient ses conditions de travail, le genre de texte qu'il doit traduire et, aussi, le type de formation qu'il a pu recevoir. Et Dieu sait que les voies sont nombreuses qui conduisent au métier de traducteur! Qui voit le traducteur voit déjà le philosophe et le poète.

Ce disant, je sais par expérience vive combien les contraintes multiples du métier et, surtout, des conditions de travail, rendent aléatoire cet idéal de la distanciation de l'objet - le texte traduit - par le sujet - le traducteur. La course au rendement, l'accumulation impitoyablement comptabilisée des mots chosifiés, les obscurités du texte original ne sont que des exemples dé cette mise en péril de la fonction et de l'art de traduire.

25. Yvon Bélaval (1973) : «Poésie», Encyclopaedia Universalis, vol. 13, p. 199, c. 3.

26. Cf. Bureau des traductions, Secrétariat d'État, La traduction au service de l'État et du pays. Doctrine, conception générale et méthode, Projet $n^{\circ} 3$, Ottawa-Hull, juillet 1978, p. 19.

27. Ibid., p. 20. 
Pour conclure, modeste mais vivante illustration de mon propos voici un poème de mon dernier recueil de poésie. Le recueil a pour titre Été d'aube ${ }^{28}$ et le poème choisi, Le mot:

\section{LE MOT}

D'un mot

A tes lèvres

Parole, mot espéré

Rêvé, mot inspiré

Pour dire le soupir

Chant troublant du mot

Eau vive, verbe créateur

Discours d'un mot

Phrase d'un son

Émoi de la voix

Résonne au creux de la terre

A l'aine de ta chair

Mot pudique

Mot unique

Emporte en un souffle

Haleine vivifiante

Et le temps et le vent

Le tourment de l'amant.

Mot, en écho

Repris, prié, crié

Invoqué

Lettres cent fois écrites

Redites, copiées

De ta main tracées

Gravées au creux

Au feu de ta chair.

Qui lira le chiffre

L'inquiétant mystère

Qui épellera,

Avec moi conjuguera

Le mot?

28. Jacques Flamand (1980): Été d'aube. Poèmes, «Création», Éditions Naaman, Sherbrooke, $112 \mathrm{p}$. 